\title{
Multiple Preemptive EDCA for Emergency Medium Access Control in Distributed WLANs
}

\author{
Shuaib K. Memon, Nurul I. Sarkar, Adnan Al-Anbuky \\ Auckland University of Technology, Private Bag 92006, Auckland 1142, New Zealand \\ Email: nurul.sarkar@aut.ac.nz
}

\begin{abstract}
The increasingly use of wireless local area networks (WLANs) in public safety and emergency network services demands for a strict quality of service (QoS) guarantee especially a large number of users report an emergency for immediate channel access. Unfortunately, the traditional IEEE 802.11e-based enhanced distributed channel access (EDCA) does not support a strict QoS guarantee for life saving emergency traffic under high loads. Previous studies have attempted to enhance the performance of EDCA called the Channel Preemtive EDCA (CP-EDCA) which is a promising idea to support emergency traffic in WLANs. However, CP-EDCA supports a single emergency traffic only (i.e. no emergency service differentiation) with high delays for increased traffic loads. To overcome this problem, we propose a class of EDCA protocol called Multiple Preemption EDCA (MP-EDCA) as a candidate to support multiple emergency traffics under high loads. Each MP-EDCA node can support up to four emergency traffics (life, health, property and environment) with different priorities in addition to support background (nonemergency) traffic. The proposed protocol privileged the high priority life-saving emergency traffic to preempt the services of low priority ones without much starvation in the network to maintain a strict QoS guarantee. The paper evaluates the performance of MP-EDCA through an extensive analysis of simulation outcome. The results obtained show that MP-EDCA outperforms CP-EDCA in achieving lower medium access control and emergency node delays.
\end{abstract}

Keywords Enhanced distributed channel access (EDCA), 802.11e, Medium access control, Quality of service (QoS)

\section{Introduction}

There has been a tremendous growth in the deployment of 802.11-based wireless local area networks 
(WLANs) [1,2]. At the same time, there is a significant growth in the use of wireless fidelity (Wi-Fi) for distributed emergency applications/services (e.g. disaster recovery, surveillance, and health monitoring) [3, 4]. These emergency applications require a strict quality of service (QoS) guarantee with the provision of in-channel preemption (e.g. channel access priority on arrival).

Fixed backbone or infrastructure based networks are highly affected by disasters such as earthquake, fire and flooding. In fact, the traditional networks such as General Packet Radio Service (GPRS), Global Systems for Mobile Communication (GSM), and other infrastructure based networks are not reliable for emergency communications (e.g. 9/11 disaster in the USA) as they quickly overloaded in the disaster affected areas even in a small urban area. However, distributed ad-hoc networks are becoming more popular for emergency networking solutions $[5,6]$.

The IEEE 802.11e working group enhanced 802.11 known as enhanced distributed channel access (EDCA) to support QoS for WLANs. The 802.11e uses EDCA mechanism to provide differentiated services to users in contention based methods. However, EDCA neither supports a strict QoS guarantee [7] nor emergency traffics [8].

To achieve a better QoS, many organizations have categorized emergency into four classes: emergency to life, health, property and environment $[9,10]$. These services can be linked to various service priorities as emergencies do not require the same level of priority. For example, emergency to life has the highest priority because nothing is more important than human lives. This is followed by prioritization to health, property and environment.

To support multiple emergency traffic and to provide a strict QoS guarantee especially to life saving emergency traffic, the traditional EDCA and its variants (e.g. CP-EDCA [11]) require improvement. Our contributions in this paper are highlighted next.

\subsection{Our contributions}

The main contribution and strength of this paper is the emphasis that emergency (traffic) service differentiation as well as multiple preemption to handle saturated emergencies (e.g. earthquake) where a large number of users demand for immediate channel access is crucial for an effective communication. This paper therefore proposes a multiple preemption EDCA (MP-EDCA) protocol, an in-channel multiple- 
preemption enhancement to CP-EDCA allowing high priority emergency frames to interrupt and replace the channel access of lower-priority ones without much starvation in the network. Our design builds on CPEDCA on the assumption that emergency services/organisations may not have the same number of resources to support dense emergencies such as tsunami, earthquake, and man-made disasters (e.g. 9/11 incident in New York) where a large number of users report emergencies. In such situations, organisations give priority to life saving traffic because nothing is more important than human life followed by health, property and environment. The most innovative aspect of this paper is the design, performance modelling, and implementation of MP-EDCA to be effective in providing a strict QoS guarantee to life saving emergencies in distributed networks. We implemented and tested MP-EDCA supporting four classes of emergency traffic (life, health, property and environment) per node with a total of 40 emergency nodes (10 nodes for each type of emergency) in OPNET simulation environment [12]. We also implemented CPEDCA and EDCA for comparison purposes. We contributed in modifying OPNET code (written in C++) to create a new process model (Fig. 5) and an emergency node model (Fig. 6) supporting multiple emergency in WLANs. We use short inter-frame spacing (SIFS) and slot-time for traffic prioritisation for simplicity in operation and compatibility with the standard EDCA and its variant CP-EDCA. To the best of our knowledge, the proposed MP-EDCA is a first piece of work contributing to the body of knowledge in the field of strict QoS and multiple emergency traffics in distributed WLANs.

\subsection{The 802.11e (EDCA) and related work}

The 802.11e is an enhanced version of the 802.11 MAC protocol to support QoS. This standard introduced two coordination functions namely, Hybrid Coordination Function Control Channel Access (HCCA) and a mandatory Enhanced Distributed Channel Access (EDCA). HCCA is a centralized control method that can support a strict QoS but the system has not been implemented widely as a result of high system complexity and inefficiency for sporadic emergency traffics [13]. In contrast, EDCA provides a quicker channel access as well as scalable to operate in ad-hoc mode making an attractive solution for achieving QoS in distributed in WLANs. 
Preemption is a good strategy to guarantee an immediate channel access for emergency traffics. Recently IEEE 802.11u-2011 [8] working group introduced a new standard called 802.11u for interworking with external networks supporting emergency traffic and preemption over infrastructure-based WLANs.

Conte et al. [14] investigated a centrally controlled admission control (AC) mechanism for 802.11e to enable emergency calls. However, it requires additional information to identify and differentiate the requests related to emergency calls. Two approaches are proposed; one to include an Emergency flag in the traffic specification field (TSpec) and the other to introduce a class of high priority emergency traffic called Traffic Stream Information (TSpec) with the source message.

Lu-min et al. [15] evaluated the performance of EDCA by considering emergency traffic in congestion. The authors have proposed an admission control method for emergency traffic. High priority emergency traffic can access the channel on arrival. Sheu, et al. [16] proposed a centralized channel preemption technique which is not suitable for distributed ad-hoc networks. Eiager et al. [17] proposed another class of preemption called a latency-aware service opportunity (LASO) where a high-class traffic curtails the transmission duration of lower class traffic. However, LASO does not allow interruption once the channel is acquired for pre-allocated time duration.

Balakrishnan et al. [11] introduced a class of preemptive channel access method called Channel Preemptive EDCA (CP-EDCA) to support emergency traffic. However, CP-EDCA does not support "multiple-emergency" traffic and is not suitable for application requiring dense emergency networking. This is because CP-EDCA treats all emergency traffic in a similar way (i.e. no emergency service differentiation) and therefore life-saving emergency traffic is highly affected. To overcome the limitations of CP-EDCA, we propose a class of EDCA protocol called Multiple-Preemptive EDCA (MP-EDCA) which is discussed in more details in Section 2.

Figure 1 illustrates the key concept and design principle of four channel access methods namely, 802.11 Distributed Coordination Function (DCF) [18], 802.11e (EDCA) [13], CP-EDCA [11], and our proposed MP-EDCA. It is obvious that the IEEE 802.11 (DCF) does not provide any service differentiation (i.e., no QoS) at all. The 802.11e (EDCA) supports voice, video, best effort, and background traffic through four queues as defined in the standard [19]. However, there is no provision for supporting emergency traffic in EDCA. 


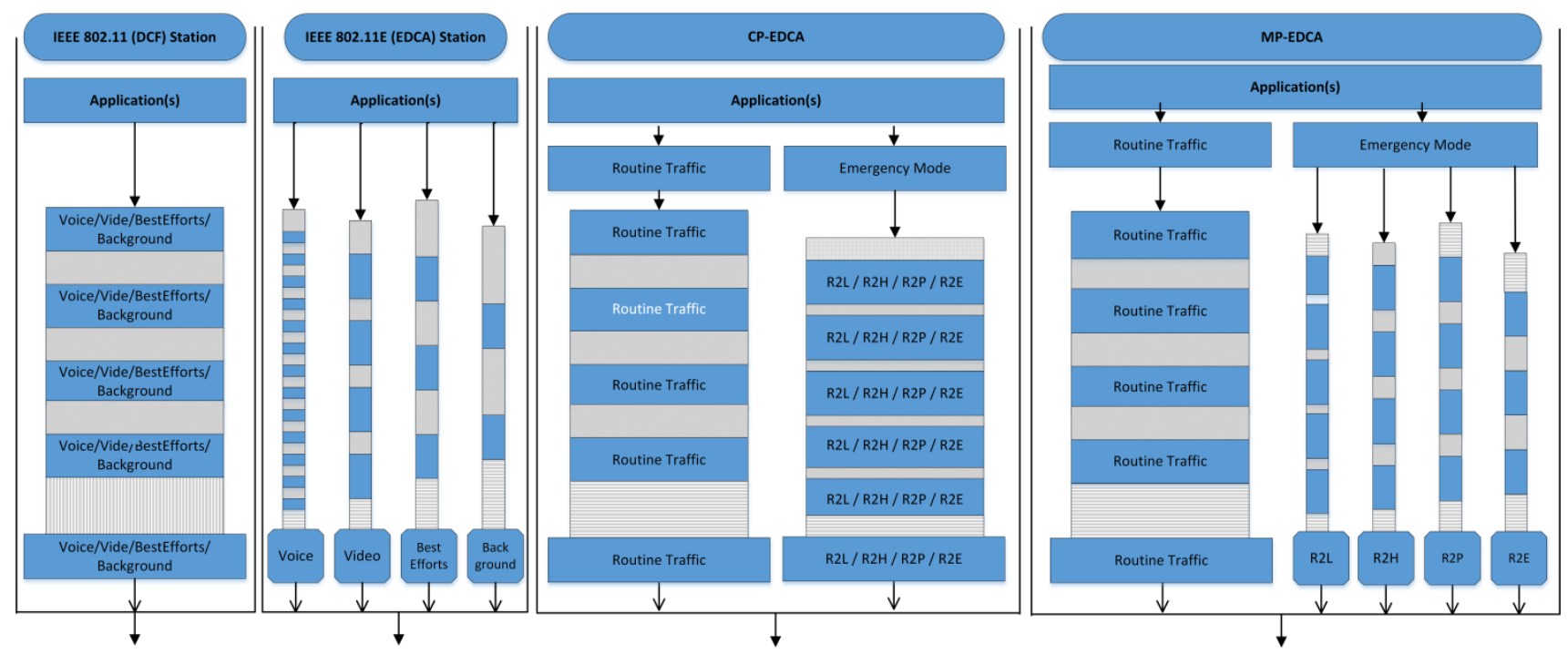

Fig. 1 Illustrating the principle of operation of 802.11 (DCF), 802.11e (EDCA), CP-EDCA and MPEDCA [11, 20, 21]

As shown in Fig. 1, CP-EDCA supports only one class of emergency traffic through a single queue in addition to serving routine traffic. The emergency traffic preempts the on-going routine traffic to acquire the channel. In contrast, the proposed MP-EDCA supports four classes (Class 1 to 4 ) of emergency traffic (per node) through four emergency priority queues. The background traffics are served through a separate queue. For instance, Class 1 (Risk to Life) emergency traffic stream may have the highest priority, followed by Class 2 (Risk to Health), Class 3 (Risk to Property), and Class 4 (Risk to Environment). Each high priority emergency traffic stream can preempt the low priority one in order to acquire the channel.

The question may arise about four classes of emergency traffics in MP-EDCA; why not implement three or five classes. We consider four classes of emergency traffics based on published literature that most organizations have categorized emergency into four classes, namely life, health, property and environment $[9,10]$. These four classes linked to services that have practical implications in real-life scenarios. For instance, life-saving emergency has the highest priority because nothing is more important than human lives. This is followed by service prioritization to health, property and environment.

It should be noted that the proposed MP-EDCA cannot support unlimited priority classes due to SIFS and slot-time constraints. The MP-EDCA approach is discussed next. 


\section{The MP-EDCA approach}

In this section we first discuss the motivation for designing MP-EDCA and then focus on protocol design strategy including the implementation aspect of process and emergency node models.

\subsection{Motivation for designer MP-EDCA}

Clearly, Balakrishnan's CP-EDCA [11] has several limitations. Firstly, it lacks service differentiation for emergency traffic (i.e. all emergencies treated the same). Secondly, CP-EDCA does not support multiple preemptions in saturated emergency situation where a large number of users demand for concurrent channel access. Consequently, the performance of CP-EDCA degrades significantly for a network with large number of users report an emergency. The proposed MP-EDCA overcomes the above limitations of CP-EDCA.

However, the main motivation for designing MP-EDCA protocol was to provide a certain QoS guarantees for all users in the network especially for life saving emergency nodes in dense emergency situation. Each MP-EDCA node supports up to four emergency traffics with different priorities in addition to support the routine/background (non-emergency) traffic without introducing starvation in the network.

\subsection{Protocol designer strategy}

MP-EDCA extends CP-EDCA by incorporating the key concept of in-channel multiple preemptions to support dense emergency traffic in distributed networks. It provides higher priority to life saving emergency than emergency to health, property and environment by carefully adjusting SIFS and slot-time in the emergency frames. Moreover, MP-EDCA employs prioritized queuing contention mechanism and contention free bursting (CFB) to allow contiguous transmission of multiple high priority emergency frames without contending the transmit opportunity (TXOP) period. Figure 2 illustrates MP-EDCA's approach of implementing in-channel multiple preemptions operating in both normal (background) traffic (Fig. 2a) and emergency traffic modes (Fig. 2b). 


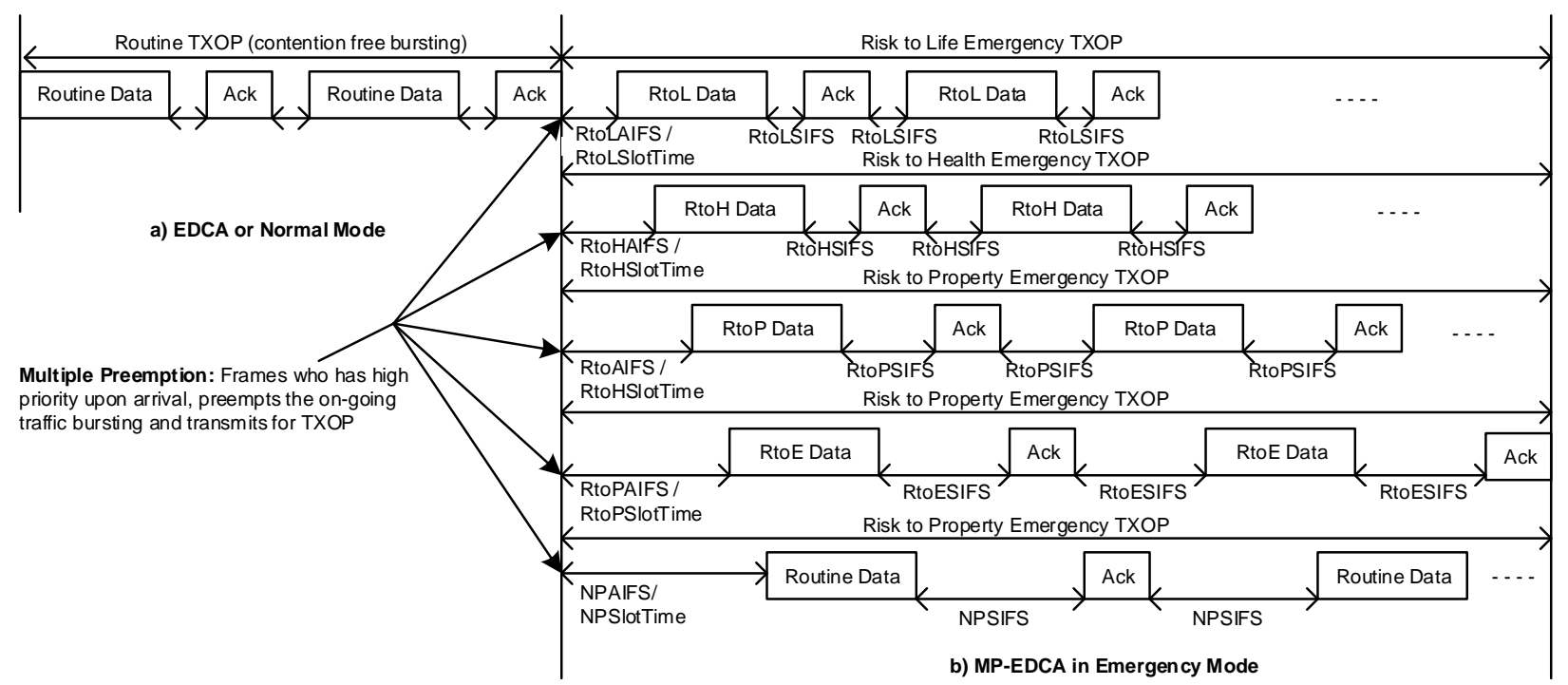

Fig. 2 Channel TXOP multiple preemptions: (a) EDCA or normal operating mode; and (b) MP-EDCA emergency mode of operation

A high priority emergency queue has privilege to interrupt on-going TXOP burst of other lowerpriority emergency (or non-emergency) queues. The interrupted queue backs off and contends after the high priority emergency bursting. The IFS and slot-time [21] of standard EDCA are modified to support multiple preemptions for the transmission of emergency frames. The MP-EDCA algorithm as well as timing sequences are highlighted for 802.11 radios next.

1) Class 1 Priority: Risk to Life SIFS (RtoLSIFS) is set to $10 \mu \mathrm{s}$ similar to standard SIFS. This minimum duration is required for PHY/MAC processing and Rx/Tx turnaround time. In each Class 1 frame burst, frames are separated by RtoLSIFS. Risk to Life (RtoL) frame burst is split by RtoLSlotTime that cannot be interrupted.

2) RtoLSlotTime slot time $(25 \mu \mathrm{s})$ is a combined period of RtoLSIFS and a Clear Channel Assessment (CCA). The slot time for Risk to Life priority is shorter than all other slot times for priorities. A CCA period is required to detect the frame in the wireless medium after the transmission initiation by another node. In MP-EDCA, the AIFS [20] duration for life saving emergency priority is one RtoLSlotTime. 
3) Class 2 Priority: Risk to Health SIFS (RtoHSIFS) is set to $25 \mu$ s which is a combined period of RtoLSIFS and CCA. In RtoL TXOP frame burst, frames are separated by RtoHSIFS. A RtoHSIFS slot time is equivalent to RtoLSIFS allowing life-saving emergency traffic to interrupt on-going Risk to Health (RtoH) priority frame burst.

4) RtoHSlotTime: This slot time (40 $\mu \mathrm{s})$ is a combined period of RtoHSIFS and a CCA. The slot time for RtoH priority is different from RtoLSlotTime. The node with RtoH priority can sense the channel for the duration of RtoHSIFS as long as there is no on-going transmission of RtoL and the channel is idle for CCA to initiate the transmission. In MP-EDCA, the AIFS [20] duration for health saving emergency priority is one RtoHSlotTime.

5) Class 3 Priority: Risk to Property SIFS (RtoPSIFS) $(40 \mu \mathrm{s})$ is a combined period of RtoPSIFS and a CCA. The RtoHSIFS separates the frames in TXOP frame burst of Risk to Property (RtoP) node. As RtoPSIFS is identical to RtoHSlotTime, allowing the lifesaving and health saving emergency traffics to interrupt an on-going transmission of RtoP nodes.

6) Risk to Property Slot Time (RtoPSlotTime): This slot time $(55 \mu \mathrm{s})$ is the combined period of RtoPSIFS and CCA. RtoPSlotTime differs from RtoLSlotTime, RtoHSlotTime, and the normal priority slot times. The node with RtoP priority can sense the channel for the duration of RtoPSlotTime to initiate the transmission as long as there is no on-going transmission from RtoL and RtoH, and the channel is idle for a period of CCA.

7) Class 4 Priority: Risk to Environment SIFS (RtoESIFS) is set to $55 \mu$ s which is a combination of RtoPSIFS and a CCA. The RtoESIFS separates the frames in TXOP frame burst of Risk to Environment (RtoE) node. The duration of RtoESIFS is identical to RtoPSlotTime, allowing RtoL, RtoH, and RtoP nodes to preempt the on-going transmission of RtoE nodes.

8) Risk to Environment Slot Time (RtoESlotTime): This slot time (70) $\mu$ s is a combined period of RtoESIFS and a CCA which is different from RtoLSlotTime, RtoHSlotTime, RtoPSlotTime, and a normal slot time. The node with a Class 4 priority can sense the channel for the duration of RtoESlottime to initiate transmissions as long as there is no on-going transmission from RtoL, RtoH, and RtoP and the channel is idle for a period of CCA. 
9) Normal Priority SIFS (NPSIFS): The 70 $\mu$ s period is a combined period of RtoESIFS and a CCA. Basically, RtoESIFS separates the frames in TXOP frame burst of normal priority (non-emergency) node. The NPSIFS is identical to RtoESlotTime, allowing RtoL, RtoH, RtoP, RtoE nodes to interrupt an on-going transmission of normal priority node.

10) Normal Priority Slot Time (NPSlotTime): This slot time $(85 \mu \mathrm{s})$ is a combined period of NPSIFS and a CCA. The nodes with no priority will sense the channel to initiate the transmission as long as the channel is not being used by any emergency nodes for the duration of a CCA period. This allows nodes to transmit background traffic in order to bring a level of fairness and thus avoiding a complete starvation in the network. This is one of the key features of MP-EDCA.

The channel access timing including SIFS and slot-time format of MP-EDCA is shown in Fig. 3. The concept is similar to IFS of $802.11 \mathrm{e}$ (EDCA) that uses the waiting time to allow high priority traffic to access the channel. An on-going frame burst sequence (Data-Ack-Data) is managed by SIFS. The smaller SIFS is set for high priority traffic. However, in MP-EDCA, the highest priority emergency traffic controls the channel by interrupting the on-going lower priority traffic burst.

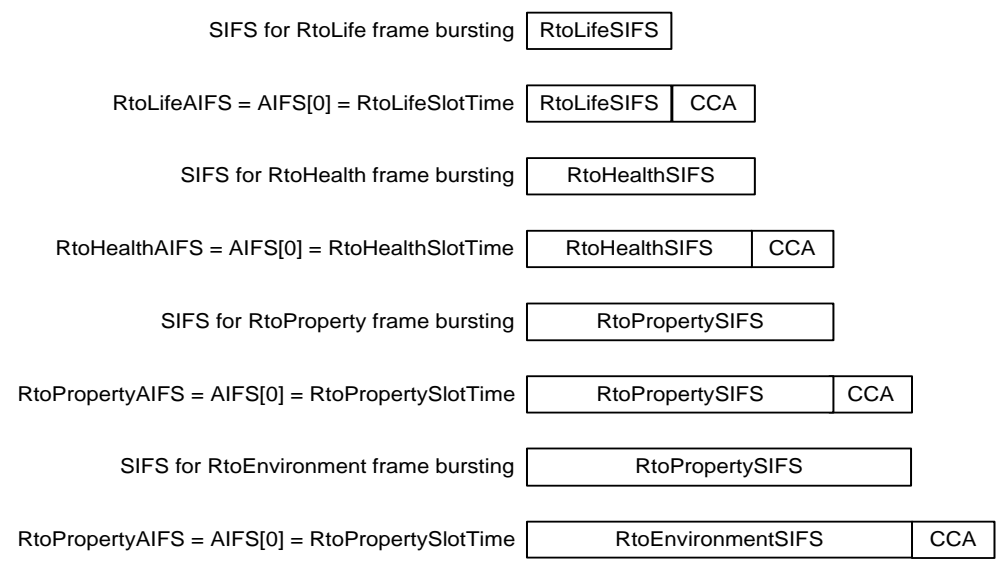

Fig. 3 MP-EDCA SIFS and slot-timing 


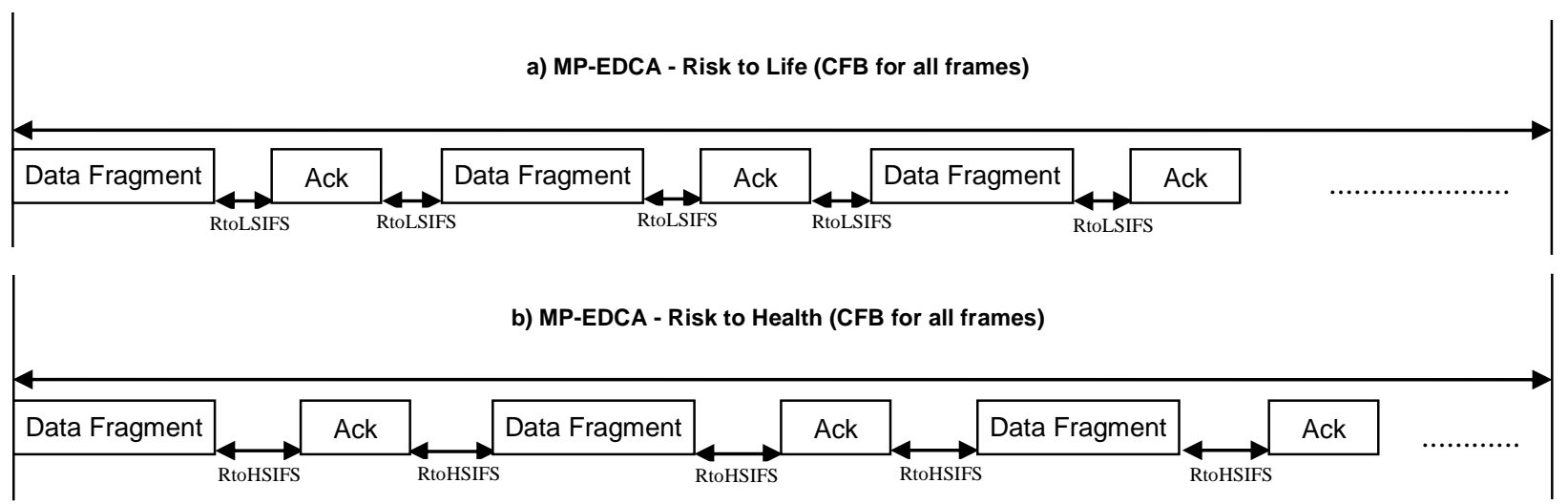

c) MP-EDCA - Risk to Property (CFB for all frames)
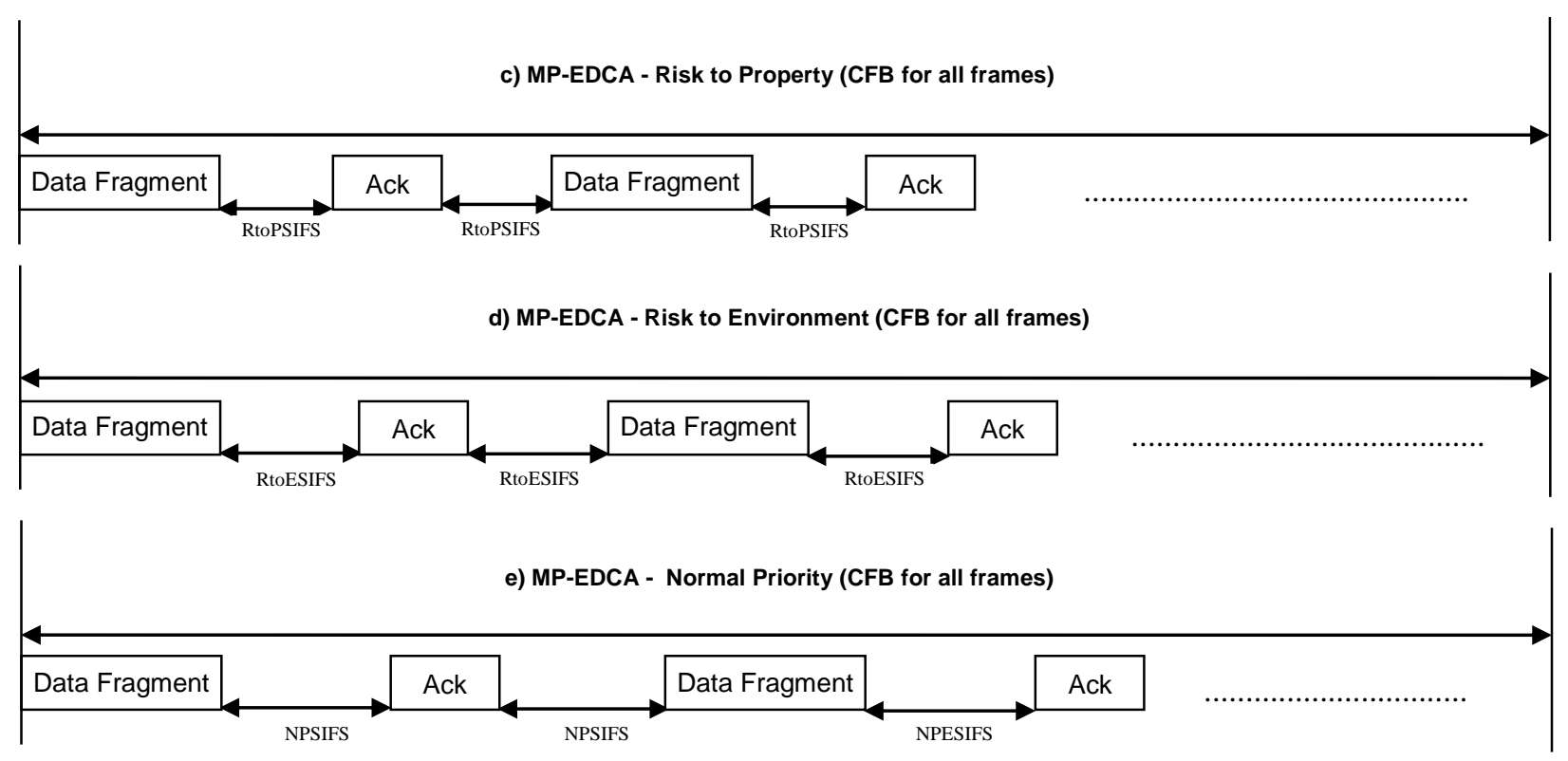

Fig. 4 Example of MP-EDCA frame bursting for emergency (a to d) and non-emergency traffic (e)

Figure 4 shows the MP-EDCA frame bursting for both emergency and normal/non-emergency traffic.

The burst for Risk to Life frames is shown in Fig. 4(a) where each frame is separated by RtoLSIFS. The frame bursts for risk to health, property, and environment are shown in Figs. 4(b), 4(c), and 4(d), respectively. Each emergency frame is identified by its own SIFS. The frame burst for a normal priority (non-emergency) is shown in Fig. 4(e).

The Risk to Life emergency TXOP burst has the highest priority (RtoLifeAIFS) followed by Risk to Health emergency, Risk to Property, and Risk to Environment frame burst. Each IFS time is separated by a CCA period, which is the minimum time required to detect a new transmission on the channel. The IFS and slot prioritization mechanisms are exclusively used in providing multiple preemption privileges to various emergency traffics. Thus, all priorities other than emergency will fall in the normal/routine category in the 
context of channel preemptions. However, prioritized channel access still exists between all traffic categories because MP-EDCA adopts the same contention mechanism as the standard EDCA. This feature allows MP-EDCA to avoid complete starvation in supporting multiple emergency traffics in the network.

\subsection{Process and emergency node models}

Figure 5 shows the MP-EDCA process model which is developed by modifying the existing wireless LAN (WLAN) process model in OPNET Modeler 16.0 (www.opnet.com). The timing modules and preemption algorithms are also implemented in OPNET. The MP-EDCA emergency node model is shown in Fig. 6, enabling immediate channel access for Risk to Life emergency traffic throughout TXOP period. So, no preemptions are allowed during life-saving emergency prioritized by the shortest waiting time. The emergency attribute has values ranging from 1 to 5 to identify the various priorities. For example, ' 1 ' is for risk to life priority, ' 2 ' for risk to health, ' 3 ' for risk to property, ' 4 ' for risk to environment, and ' 5 ' for normal (non-emergency) priority.

New Risk to Life emergency frames are transmitted if the channel is sensed idle for a period of EPAIFS/RtoLSlotTime which is the smallest time slot. Similarly, high priority emergency frames are transmitted if the channel is sensed idle for a SlotTime. A high priority emergency frame has smaller SlotTime than the low priority emergency ones; therefore high priority emergency frames can break the ongoing lower-priority emergency transmissions on arrival. On the next round, high priority emergency frames wait for a shorter period (slot-time) to access the channel. The low priority emergency frames back off and contend the channel until the high priority emergency burst finished transmission.

Network allocation vector (NAV) is used to protect the entire burst of frames subject to the TXOP limit [20]. The MP-EDCA complies with 802.11e standard by allowing multiple-preemptions even though NAV is set. However, NAV duration is set to protect a single frame with immediate acknowledgement only. 


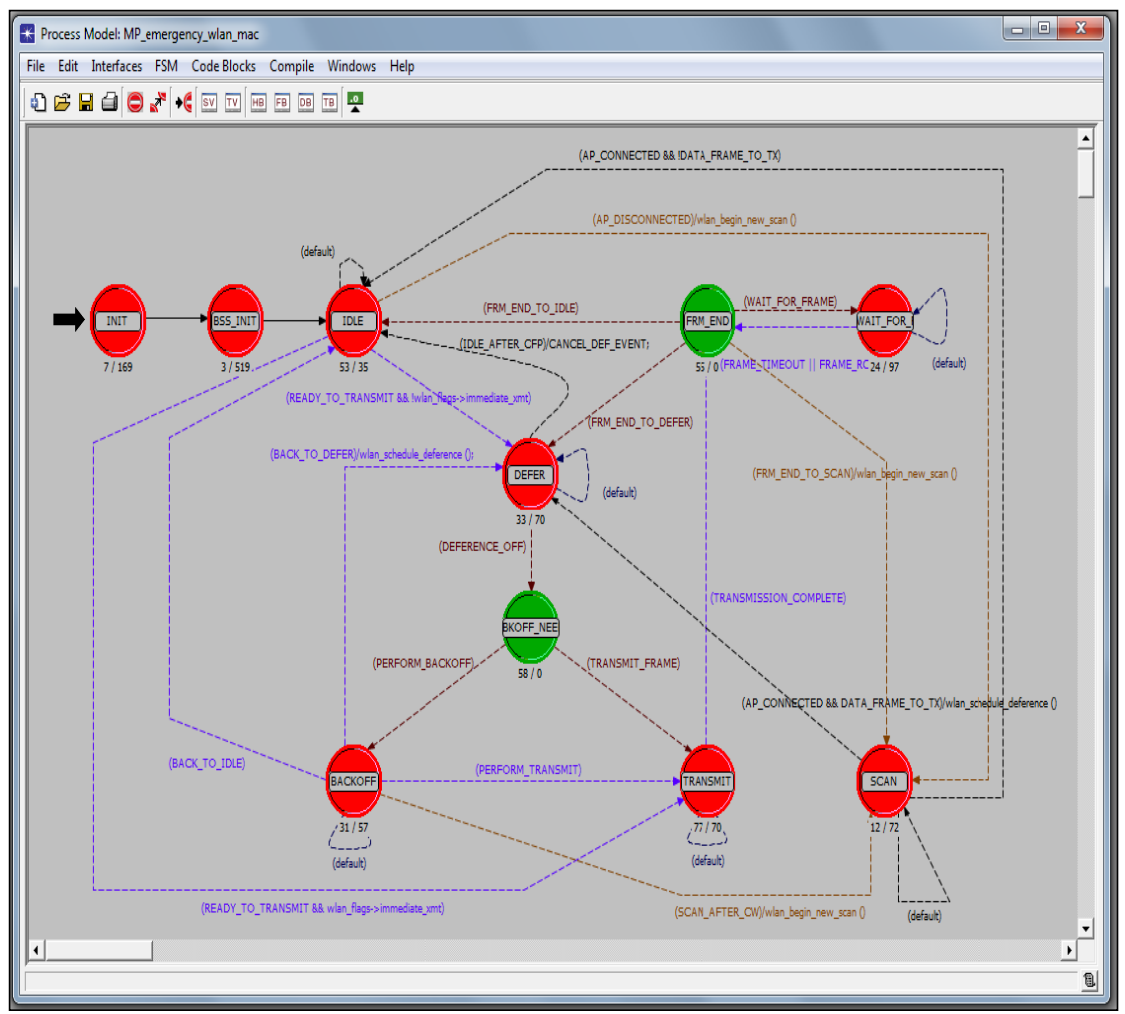

Fig. 5 MP-EDCA Process model 


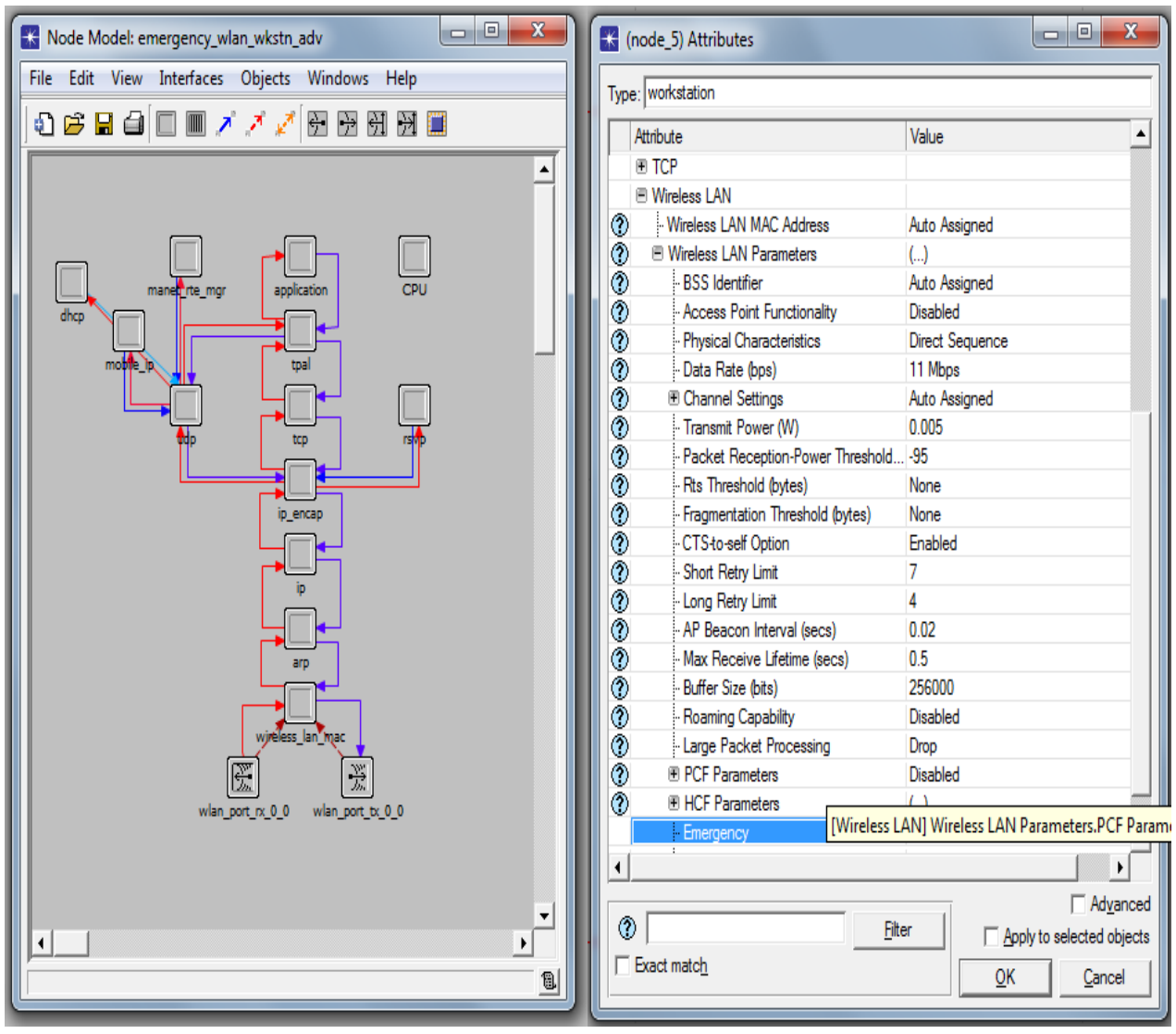

Fig. 6 MP-EDCA Emergency node model

The proposed MP-EDCA achieves both in-node and inter-node multiple preemptions (i.e. multiple interruptions of lower-priority TXOP of other nodes) by allowing IFS and slot-times of all priority queues of each node on the network. We use the standard 802.11e (EDCA) contention procedure and priority queuing (channel bursting) mechanisms to make compatible with the existing EDCA protocol and it's variants [20].

\section{Performance study and simulation setup}

To study the performance of the proposed MP-EDCA and to compare it with the standard EDCA [20] and Balakrishnan's CP-EDCA [11], we developed OPNET-based simulation models [22]. The OPNET simulation tool was chosen due to its popularity and credibility [23]. 


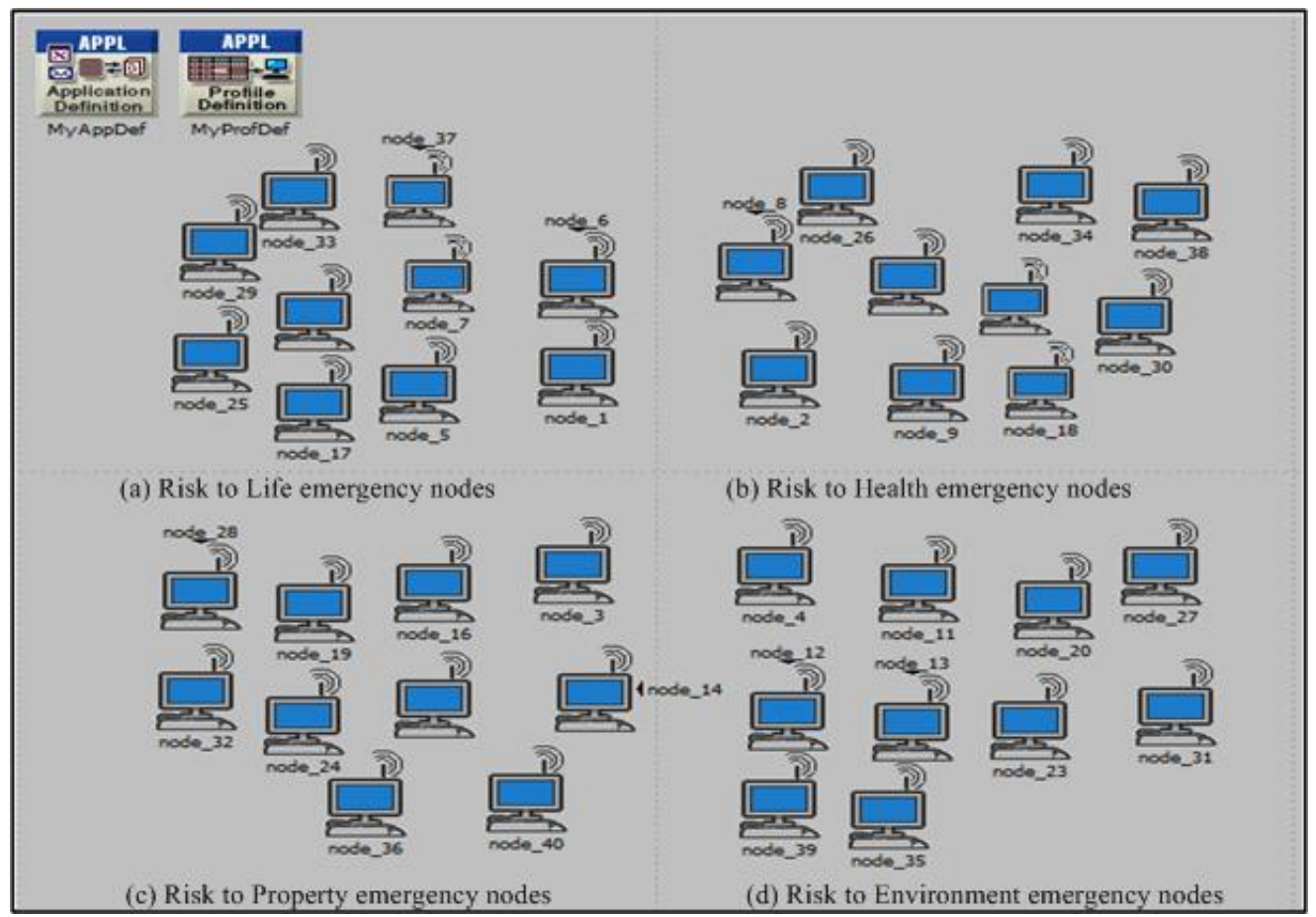

Fig. 7 OPNET represntation of fully connected MP-EDCA network with $\mathrm{N}=40$ emergency nodes

Figure 7 shows the MP-EDCA network model with $\mathrm{N}=40$ emergency nodes. It consists of 4 sub models with identical configuration (10 emergency nodes per model). For example, Fig. 7(a) represents risk to life emergency (Class 1). The risk to health (Class 2), risk to property (Class 3 ), and risk to environment (Class 4) are shown in Fig. 7(b), (c), and (d), respectively. Each MP-EDCA node supports up to four classes (Class 1 to 4 ) of emergency traffic with different priorities (uniform node distribution). 
Table 1 MAC parameters used in simulation

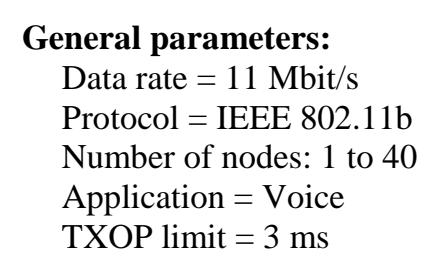

\section{Contention parameters:}

\section{a) MP-EDCA}

\begin{tabular}{l} 
Risk to Life \\
\hline RtoLSIFS $=10$ \\
RtoLSlotTime $=25$ \\
AIFS $[0]=1$ slot \\
WMin[0] = 2 slots \\
WMax[8] = 8 slots
\end{tabular}

b) CP-EDCA

\begin{tabular}{l|} 
Class 0 (Emergency Priority) \\
\hline EPSIFS $=10$ \\
EPSlotTime $=25$ \\
AIFS $[0]=1$ slot \\
WMin $[0]=2$ slots \\
WMax $[8]=8$ slots \\
c) $\quad$ EDCA \\
SIFS $=10 \mu$ s for all priorities \\
Slot Time $=20 \mu$ s (default) for all priorities \\
AIFS $[2]$ \\
Wmin $[0]=2$ slots \\
WMax $=8$ slots
\end{tabular}

Class 1 (Normal Priority)

NPSIFS $=40$

NPSlotTime $=55$

AIFS[1] $=4$ slots

WMin[1] $=8$ slots

WMax [1] = 64 slots

\begin{tabular}{l} 
Risk to Environment \\
\hline RtoPSIFS $=55$ \\
RtoESlotTime $=70$ \\
AIFS $[0]=1$ slot \\
WMin $[0]=2$ slots \\
WMax $[8]=8$ slots
\end{tabular}




\section{Results and comparison}

We present the results of investigation for four emergency classes namely, Risk to Life ("Class 1"), Risk to Health ("Class 2"), Risk to Property ("Class 3"), and Risk to Environment ("Class 4"). The performance of MP-EDCA is evaluated by extensive simulation experiments under high traffic loads. We simulated 30 Scenarios; 10 for each of EDCA, CP-EDCA and MP-EDCA. The simulation results report the steady-state behavior of the network and have been obtained with the relative error of $<1 \%$, at the $99 \%$ confidence level.

The MAC packet delay (network-wide as well as individual node) is one of the key performance metrics we consider in this paper. The delay is measured (in sec) from the moment a frame is queued at the MAC-layer until the frame is successfully transmitted. This includes channel contention, queuing, and frame transmission time.

Figure 8 shows the average MAC delay of MP-EDCA, EDCA and CP-EDCA with varying node density for $\mathrm{N}=4$ to 40 nodes. The MAC delays are similar for all three schemes (MP-EDCA, EDCA and CPEDCA) for $\mathrm{N}=4$ to 16 nodes. However, the average delays for both EDCA and CP-EDCA rise sharply for $\mathrm{N}=16$ to 40 nodes. One can observe that MP-EDCA offers lower packet delays than both EDCA and CPEDCA for $\mathrm{N}>16$ nodes. For instance, for $\mathrm{N}=40$ nodes, the network-wide MAC delays for MP-EDCA, EDCA, and CP-EDCA are $3.4 \mathrm{sec}, 8.7 \mathrm{sec}$, and $8.5 \mathrm{sec}$, respectively. We found that MP-EDCA achieves about $60 \%$ lower packet delays than CP-EDCA. Considering $11 \mathrm{Mbit} / \mathrm{s}$ channel, about $5 \mathrm{sec}$ reduction in one-hop MAC delay per emergency frame can be achieved using MP-EDCA, is a significant improvement.

Now let us focus on the performance of MP-EDCA for $\mathrm{N}=16$ to 40 emergency nodes. The question may arise about the lowest MAC delays for nodes 28 and 32 in the network. For example, the networkwide average MAC delays for $\mathrm{N}=28$ and 32 emergency nodes are $1.1 \mathrm{sec}$ and $1.09 \mathrm{sec}$, respectively. Recall that each MP-EDCA node can support up to four emergency traffics with different priorities. Perhaps serving more high priority emergency traffics (e.g. risk to life) at nodes 28 and 32, contributed to lower average MAC delays.

The main conclusion is that (Fig. 8) the network-wide MP-EDCA's MAC delay is significantly better (i.e. smaller) than that of CP-EDCA, especially under medium-to-high traffic loads. 


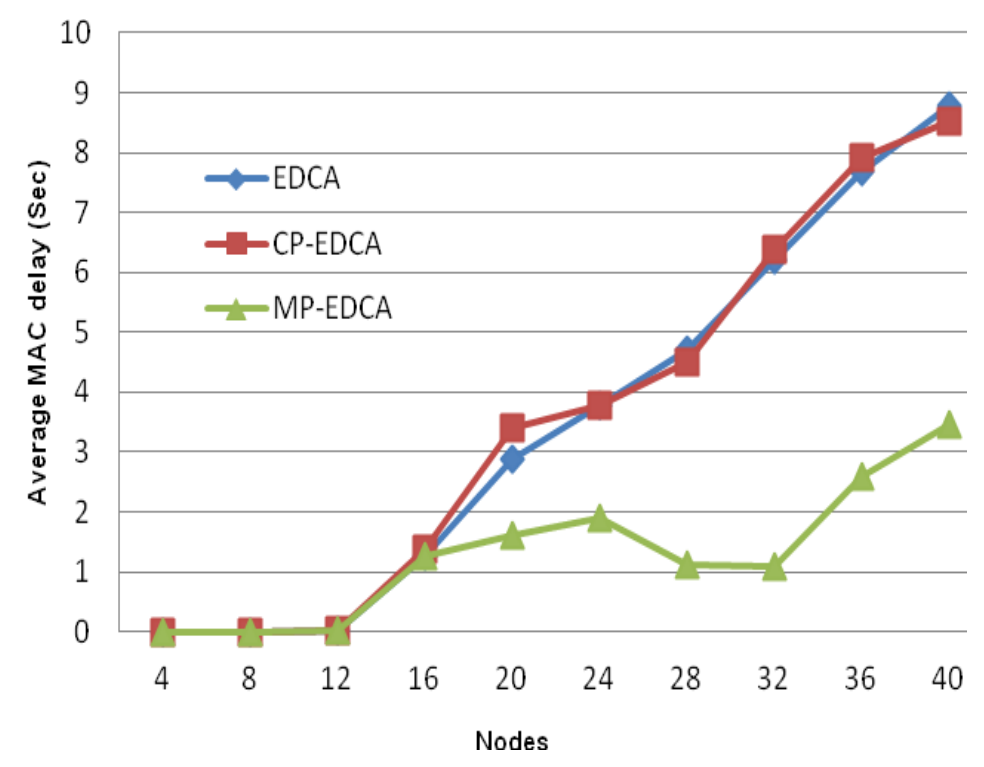

Fig. 8 Network-wide MAC delay of EDCA, CP-EDCA and MP-EDCA

An average MAC delay might not be an accurate measures for Class 1 (Risk to Life) node because the low priority emergency traffic may experience slightly higher delays compared to high priority emergency traffics.

To analyse the complete delay characteristics, we plot a single node average MAC delay against node density in Fig. 9. We observe that the MAC delay of MP-EDCA Class 1 emergency node is significantly better (i.e. lower) than the single emergency node of CP-EDCA and a single node (non-emergency) delay of EDCA for $\mathrm{N}>12$ nodes.

By comparing EDCA and CP-EDCA we observe that EDCA performs slightly better than CP-EDCA in terms of achieving lower MAC delays for $\mathrm{N}>12$ nodes. This is not a surprising result because we are basically comparing a single node MAC delay for two different things; an emergency node in CP-EDCA and a non-emergency node in EDCA. However, the increased MAC delays for CP-EDCA could be due to protocol strategy where an emergency traffic is supported at the price of increased delays. 


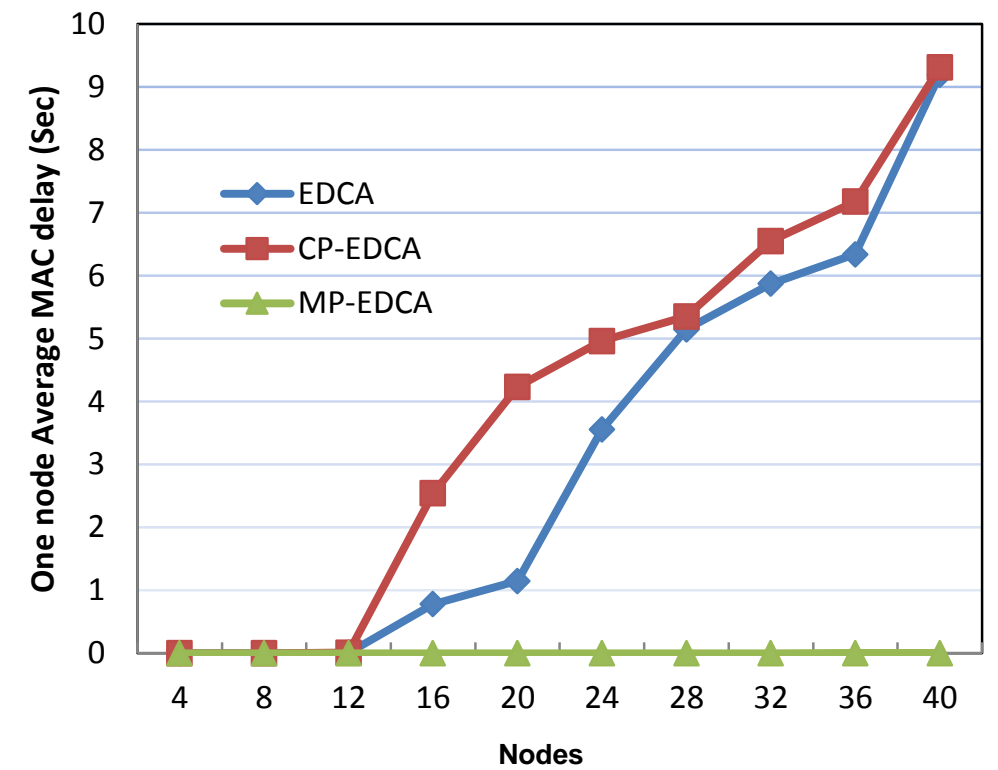

Fig. 9 MAC delay for a Class 1 (Risk to Life) emergency node

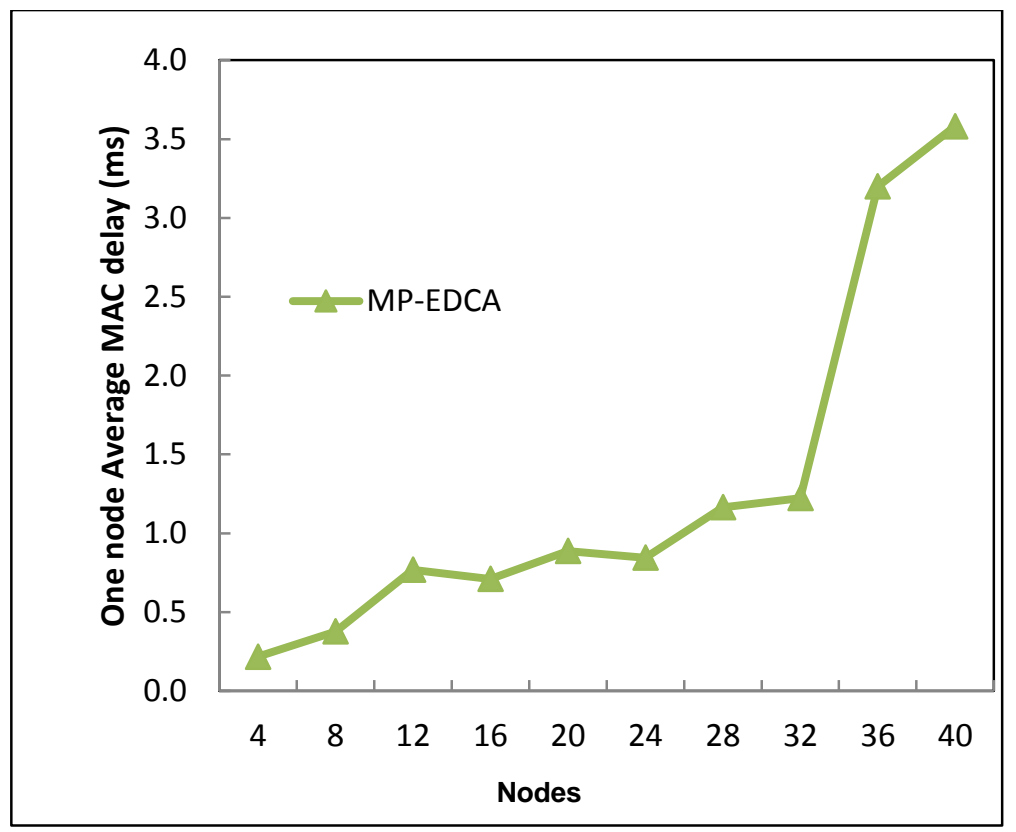

Fig. 10 The average MAC delay of MP-EDCA for Class 1 emergency node

To quantify the performance gain of MP-EDCA, a single Class 1 node MAC delay is redrawn in Fig. 10. We observe that the average MAC delays for MP-EDCA is less than $1.5 \mathrm{~ms}$ for up to 32 emergency nodes. A slight increase in MAC delay is observed for $\mathrm{N}>32$ nodes, but this increase is not very 
significant. In fact, MP-EDCA achieves just below $4 \mathrm{~ms}$ at $\mathrm{N}=40$ nodes. For example, one Risk to Life node MAC delay of MP-EDCA, one emergency node delay of CP-EDCA (as all emergencies are treated equally) and a single node delay of EDCA (non-emergency traffic) are $3.6 \mathrm{~ms}, 9.31 \mathrm{sec}$, and $9.19 \mathrm{sec}$, respectively at $\mathrm{N}=40$ nodes.

The main conclusion is that (Figs. 8 and 9) MP-EDCA outperforms both EDCA and CP-EDCA for medium to high traffic loads, especially $\mathrm{N}>12$ nodes. One can observe that the Class 1 emergency node of MP-EDCA achieved about $99.9 \%$ lower delays than CP-EDCA. The lowest possible channel access delays for risk to life emergency traffic in dense emergency is a significant achievement offered by MP-EDCA.

\section{Conclusion and future work}

In this paper we proposed an in-channel multiple service preemptions protocol called MP-EDCA to provide immediate channel access privileges to high priority emergency traffic in distributed networks. MP-EDCA provides exclusive medium access precedence for high priority emergency traffic in saturated emergency without much starvation in the network which is a significant QoS improvement to 802.11e (EDCA) and its variants CP-EDCA. The performance of MP-EDCA is evaluated by extensive simulation. Results have shown that the proposed MP-EDCA outperforms both Balakrishnan's CP-EDCA [11] and the existing EDCA. For instance, MP-EDCA achieved up to $60 \%$ lower MAC delays (network-wide) and about $99 \%$ lower delays for a single emergency node than CP-EDCA. The proposed MP-EDCA can provide QoS guarantees to emergency nodes under high traffic conditions more effectively. Furthermore, immediate channel access for life saving emergency traffic is guaranteed even in saturated emergency for nodes with

emergency exist. However, when all nodes are in 'risk to life' (an unusual case), MP-EDCA performs as good as the CP-EDCA. The performance of MP-EDCA in noisy channel is suggested as an extension of the work presented here. 


\section{References}

1. Mulhanga, M.M., S. Rito Lima, and P. Carvalho, Characterising University WLANs within Eduroam Context, in Smart Spaces and Next Generation Wired/Wireless Networking. 2011. p. 382394.

2. Shorey, R., A. Ananda, and W.T. Oai, Mobile, wireless, and sensor networks: technology, applications, and future directions. 1st ed, ed. I. press. 2006, Hoboken, New Jersey: IEEE Press Wiley.

3. Rashvand, H.F., et al., Ubiquitous wireless telemedicine. IET Communications 2008. 2(2):237-254

4. Chiti, F., et al., A broadband wireless communications system for emergency management. IEEE Wireless Communications, 2008. 15(3):8-14.

5. Graaf de, M., et al. Easy Wireless: Broadband ad-hoc networking for emergency service. in the Sixth Annual Mediterranean Ad Hoc Networking Workshop. 2007. Corfu, Greece June 12-15, pp. 32-39.

6. Yong, B., et al. Emergency communication system by heterogeneous wireless networking. in the IEEE International Conference on Wireless Communications, Networking and Information Security (WCNIS). 2010 June 25-27, pp. 488-492.

7. Abbas, A.M. and K. Al Soufy, Saturation Analysis of IEEE 802.11 EDCA for Ad Hoc Networks, in Contemporary Computing, M. Parashar, et al., Editors. 2012, Springer Berlin Heidelberg. p. 419430.

8. IEEE Std. 802.11u-2011, IEEE Standard for Information Technology - Telecommunications and information exchange between systems - local and metropolitan area networks - specific requirements - part:11: Wireless Medium Access Control (MAC) and Physical Layer (Phy) Specifications, Amendment 9: Interworking with External Networks 2011: New York.

9. Leathrum, J.F. (2003) Quine McCluskey Tabular Minimization Method. Retrieved 20 September, from http://www.ece.odu.edu/ leathrum/ECE241_284/support/quine.html

10. Whitten, J.L., L.D. Bently, and K.C. Dttman, Systems analysis and design methods. 5th ed. 2001, New York: McGraw-Hill Higher Education.

11. Balakrishnan, M., et al., Channel preemptive EDCA for emergency medium access in distributed wireless networks. IEEE Transactions on Wireless Communications, 2009. 8(12):5743-5748.

12. (2016) OPNET Modeler. Retrieved January 10, 2016, from www.opnet.com

13. Lee, H., et al., A performance analysis of block ACK scheme for IEEE 802.1 le networks. Computer Networks, 2010. 54(14):2468-2481.

14. Conte, A. and P. Dauchy, Method of enabling an emergency call in an IEEE 802.11e enabled wireless local area network, U.S.P.A. Pblication, Editor. 2006: United States.

15. Lu-ming, C., et al., Performance evaluation on IEEE 802.11e considering emergency calls in congested situation. The Journal of China Universities of posts and telecommunications, 2007. 14(1):50-59.

16. Sheu, T.L., Y.J. Wu, and B. Li, A Generalized Channel Preemption Model for Multiclass Traffic in Mobile Wireless Networks. IEEE Transactions on Vehicular Technology, 2007. 56(5):2723-2732. 
17. Eiger, M., M. Elaoud, and R. RMorera, Latency aware service opportunity window based scheduling, U.S. Patent, Editor. 2008: United States.

18. IEEE 802.11 Stand. for Wireless LAN Medium Access Control (MAC) and Physical Layer (PHY) specifications. 1997: New York.

19. IEEE Std. 802.1D-2004. IEEE Standard for Local and Metropolitan Area Networks: Media Access Control (MAC) Bridges. 2004: New York.

20. IEEE Std. 802.11e-2005, IEEE Standard for Information Technology - Telecommunications and information exchange between systems - Local and metropolitan area networks - Specific requirements Part 11: Wireless LAN Medium Access Control (MAC) and Physical Layer (PHY) specifications Amendment 8: Medium Access Control (MAC) Quality of Service Enhancements. 2005: New York.

21. IEEE Std. 802.11-2007, IEEE Standard for Information Technology - Telecommunications and information exchange between systems - local and metropolitan area networks - specific requirements - part:11: Wireless Medium Access Control (MAC) and Physical Layer (Phy) Specifications, (Revision of IEEE 802.11-1999). 2007: New York.

22. OPNET Modeler. Retrieved September 7, 2015, from www.opnet.com

23. Sarkar, N.I. and J. Gutierrez, Revisiting the Issue of the Credibility of Simulation Studies in Telecommunication Networks: Highlighting the Results of a Comprehensive Survey of IEEE Publications. IEEE Communications Magazine, 2014. 52(5):218-224. 